\title{
Tradução do Convexity Assessment Guide para a língua portuguesa
}

\author{
Translation of the Convexity Assessment Guide into the Portuguese language \\ Traducción del Convexity Assessment Guide a la lengua portuguesa
}

\begin{abstract}
Bruna Prini Rafaldini1,2, Nádia Antônia Aparecida Poletti1,3, Paula Buck de Oliveira Ruiz', Regina Helena Squizatto ${ }^{1,5}$, Andressa de Oliveira Lopes ${ }^{1,6}$, Niara Carla de Oliveira ${ }^{1,7}$
\end{abstract}

ORCID IDS

Rafaldini BP (D) https://orcid.org/0000-0001-6086-8459

Poletti NA (D) https://orcid.org/0000-0001-9116-9773

Ruiz PBO (iD https://orcid.org/0000-0001-5598-6776

Squizatto RH (D) https://orcid.org/0000-0002-6060-1583

Lopes AO (iD https://orcid.org/0000-0002-9991-5838

de Oliveira NC (DD https://orcid.org/0000-0002-3191-6586

\section{HOW TO CITE}

Rafaldini BP, Poletti NAA, Ruiz PBO, Squizatto RH, Lopes AO, de Oliveira NC. Tradução do Convexity Assessment Guide para a língua portuguesa. ESTIMA, Braz. J. Enterostomal Ther., 16: e4018. https:// doi.org/10.30886/estima.v16.633 PT

\section{RESUMO}

Objetivos: Traduzir o guia Convexity Assessment Guide para a língua portuguesa e realizar sua validação semântica, linguística, conceitual e cultural. Métodos: Estudo de tradução, descritivo qualitativo, seguindo as fases preconizadas: tradução, tradução de volta à língua de origem, comparação das versões originais e da traduzida de volta ao português, validação por comitê nacional de especialistas. Resultados: Foram obtidas 400 avaliações - 49 anuladas e 351 válidas - o que representa o total utilizado para a análise de concordância dos itens. Após a análise, verificaram-se seis itens discordantes, revistos de acordo com as sugestões dos especialistas, obtendo-se, então, concordância maior de 90\% em todos os itens avaliados. Conclusão: Verificou-se alto nível de concordância do comitê de avaliadores, garantindo a continuidade do estudo com a validação transcultural do Guia de Convexidade para a realidade brasileira. Esta pesquisa promoveu a tradução de instrumento que auxilia na indicação clínica de dispositivos coletores para estomias. Obteve-se a validação da tradução para a língua portuguesa, sendo essa a primeira fase do processo de validação transcultural.

DESCRITORES: Enfermagem; Estomia; Tradução; Guia de Prática Clínica; Estomaterapia.

\footnotetext{
${ }^{1}$ Faculdade de Medicina de Rio Preto - Programa de Pós-Graduação em Enfermagem - São José do Rio Preto/SP - Brasil.

${ }^{2}$ Hollister do Brasil - Coordenação de Educação Clínica - São Paulo/SP - Brasil.

${ }^{3}$ Faculdade de Medicina de Rio Preto - Curso de Graduação em Enfermagem - Departamento de Enfermagem Geral - São José do Rio Preto/SP - Brasil.

${ }^{4}$ Universidade de São Paulo - Programa de Pós-Graduação em Gerenciamento em Enfermagem - São Paulo/SP - Brasil. 5Unimed São Jose do Rio Preto - Departamento de Enfermagem - Setor de Emergência - São José do Rio Preto/SP - Brasil.

${ }^{6}$ Grupo Empório Saúde - São José do Rio Preto/SP - Brasil.

7Unimed São Jose do Rio Preto - Departamento Unimed Lar - São José do Rio Preto/SP - Brasil.

Autor correspondente: Bruna Prini Rafaldini | Rua São Domingos, 978/101 - Vila Caiçara | CEP: 11706-380 - Praia Grande/SP

E-mail: brunaprini@yahoo.com.br

Recebido: Ago 032018 | Aceito: Dez 152018
} 


\begin{abstract}
Objectives: Translate the Convexity Assessment Guide into the Portuguese language and perform its semantic, linguistic, conceptual and cultural validation. Methods: Translation study, qualitative descriptive, following the recommended phases: translation, back translation, comparison of original and translated versions back to Portuguese, validation by national experts committee. Results: It was obtained 400 evaluations - 49 annulled and 351 valid - which represents the total used for the agreement analysis of the items. After the analysis, there were six discordant items, reviewed according to the experts' suggestions, obtaining, then, a greater agreement of $90 \%$ in all items evaluated. Conclusion: There was a high level of agreement among the evaluator's committee, ensuring the continuity of the study with the cross-cultural validation of the Convexity Guide for the Brazilian reality. This research promoted the translation of an instrument that assists in the clinical indication of collector devices for stomas. The validation of the translation into the Portuguese language was obtained, being this the first phase of the process of cross-cultural validation.
\end{abstract}

DESCRIPTORS: Nursing; Stoma; Translation; Guide to Clinical Practice; Stomatherapy.

\title{
RESUMEN
}

Objetivos: Traducir la guía Convexity Assessment Guide a la lengua portuguesa y realizar su validación semántica, lingüística, conceptual y cultural. Métodos: Estudio de traducción, descriptivo cualitativo, siguiendo las etapas recomendadas: traducción, traducción de vuelta a la lengua de origen, comparación de las versiones originales y de la traducida de vuelta al portugués, validación por parte de comité nacional de especialistas. Resultados: Fueron obtenidas 400 evaluaciones - 49 anuladas y 351 válidas - lo que representa el total utilizado para el análisis de concordancia de los elementos. Después del análisis, se verificaron seis elementos discordantes, revisados de acuerdo con las sugerencias de los especialistas, obteniendo, así, concordancia mayor a $90 \%$ en todos los elementos evaluados. Conclusión: Se verificó alto nivel de concordancia del comité de evaluadores, garantizando la continuidad del estudio con la validación transcultural de la Guía de Convexidad para la realidad brasileña. Esta investigación promovió la traducción de instrumento que ayuda en la indicación clínica de dispositivos colectores para estomas. Se obtuvo la validación de la traducción a la lengua portuguesa, siendo esa la primera etapa del proceso de validación transcultural.

DESCRIPTORES: Enfermería; Estoma; Traducción; Guía de Práctica Clínica; Estomaterapia.

\section{INTRODUÇÃO}

Atualmente, há uma lacuna, em pesquisas nacionais, sobre o tema tradução ou desenvolvimento de guias relacionados ao uso da convexidade em estomias. No entanto, esse tipo de ferramenta vem sendo utilizada com eficiência na América do Norte, como evidenciado em eventos internacionais, como no 2016 WOCN Society \& CAET Joint Conference, Montreal, Canadá1 ${ }^{1}$ e no $21^{\text {st }}$ WCET Biennal Congress, Cape Town, África do Sul ${ }^{2}$, nos quais foram apresentados estudos relevantes a respeito do desenvolvimento de ferramentas para auxílio na indicação de equipamentos coletores, como o uso da convexidade.

No Brasil, estudos a respeito da utilização da convexidade e padronização dessa linguagem ainda são poucos e vêm sendo desenvolvidos, em sua maioria, como estudos de caso, relatos de experiência e revisões bibliográficas veiculados apenas no formato de resumo em anais ${ }^{3}$. As pesquisas que visam à tradução de instrumentos de diferentes áreas assistenciais e gerenciais têm por finalidade disseminar tecnologias para aqueles que ainda não se beneficiaram com o desenvolvimento de novos diagnósticos, terapêuticas ou ainda ferramentas que instrumentalizam as tomadas de decisão. Estudos como esses são amplamente utilizados na área da saúde no Brasil, em função da escassez de literatura e, principalmente, pela complexidade envolvida na criação de instrumentos válidos no âmbito nacional e internacional ${ }^{4,5}$.

A carência de instrumentos que auxiliem na tomada de decisão a respeito dos equipamentos coletores para estomias e hábitos intestinais no Brasil estimula a busca por ferramentas já cientificamente provadas e validadas que, quando traduzidas de forma criteriosa e conforme um escopo metodológico, podem favorecer profissionais e beneficiar os pacientes com uma assistência de enfermagem segura, baseada em evidência científica ${ }^{6}$.

Com o aumento da expectativa de vida da população brasileira, identificou-se um crescimento na confecção de novas estomias e longevidade daqueles que já vivem com essa condição, tornando ainda mais crítica a necessidade de atendimento especializado e de desenvolvimento de tecnologias inovadoras 7 . As pessoas com estomias podem experimentar complicações a elas relacionadas nos diversos períodos de sua vida e, apesar do desenvolvimento das técnicas 
cirúrgicas, as taxas de complicações ainda permanecem entre 25 a $59 \%$ dos pacientes nos três primeiros meses de pós-operatório ${ }^{8}$.

Uma das complicações diagnosticadas com maior frequência é a dermatite relacionada ao contato do efluente com a pele, e o recurso mais utilizado para prevenção das dermatites periestomais é a utilização de um dispositivo coletor que se ajuste perfeitamente aos contornos abdominais e que mantenha aderência confortável e segura entre o dispositivo e a pele. Em diversas situações, a convexidade é utilizada para manter esse encaixe da barreira adesiva com a anatomia abdominal, permitindo a adesão completa do equipamento com a pele, evitando descolamento e vazamento precoce do dispositivo coletor 9 .

Em 2012, pesquisadores internacionais classificaram a convexidade como a curvatura da barreira de pele que vai ao encontro à pele periestomal. A terminologia e as características da convexidade foram classificadas de acordo com a profundidade, o perfil, a pressão e a flexibilidade. A profundidade pode ser rasa (menor que 1,5 mm), moderada (de 1,5 $\mathrm{mm}$ a 6,3 $\mathrm{mm}$ ) e profunda (acima de 6,3 $\mathrm{mm}$ ), de acordo com o perfil de inclinação, sendo suave ou acentuada, sua flexibilidade macia ou firme, sua pressão que está relacionada à tensão baixa ou alta e localização periférica ou central. A convexidade pode ser integrada à barreira já na sua construção ou customizada, de acordo com a necessidade, adicionando-se adjuvantes, como anéis planos ou convexos, auxiliando na adaptação dos equipamentos e prevenindo complicações periestomais ${ }^{9}$.

O manejo das complicações exige esforço multidisciplinar, mas principalmente do enfermeiro que lança mão de todos os dispositivos disponíveis no mercado; porém, os guias de avaliação para auxílio na indicação de diferentes tipos de dispositivos são escassos ${ }^{9}$. Os altos custos envolvidos nos cuidados com estomias podem mostrar-se extremamente elevados, principalmente nos casos de complicações periestomais, chegando ao aumento de $75 \%$ nas despesas ${ }^{8,10}$, valores que sinalizam a necessidade de melhorias nas políticas de saúde, visando à promoção da qualidade de vida e diminuição das perdas para os serviços de saúde. Entretanto esses progressos na esfera da saúde pública e privada concretizam-se por meio de evidências científicas que fomentam os gestores com dados concretos e sistematizados, possibilitando o planejamento e o gerenciamento orçamentário consciente que permita o atendimento especializado e o fornecimento de equipamentos coletores personalizados para esse grupo populacional.

Alguns estudos referentes ao manejo de estomias complicadas e também a respeito da influência de medicamentos na consistência e características do efluente indicam a convexidade em situações, como estomias retraídas, dobras de pele, uso de quimioterapia, abdome flácido, vazamentos frequentes do efluente ou ainda quando a durabilidade do equipamento não atinge o esperado pelo usuário. São essas ocorrências complexas que impactam diretamente a vida daqueles que vivem com a estomia, assim como representam grandes desafios para os profissionais de saúde e para os serviços ${ }^{11}$.

Nesse contexto, a vivência na assistência especializada em estomaterapia aponta para uma lacuna de pesquisas científicas que fundamentem decisões clínicas e gerenciais. Dessa forma, o interesse pela realização desta pesquisa emergiu dessa ausência de ferramentas que fundamentem a tomada de decisão do enfermeiro na atuação ante a pessoa com estomia e na eleição do equipamento coletor ideal para cada paciente em cada momento de sua vida. Portanto, a tradução do guia Convexity Assessment Guide para a língua portuguesa brasileira torna-se importante como primeiro passo para o desenvolvimento de um instrumento validado para a realidade brasileira, uma vez que a convexidade ainda não é estudada com profundidade no Brasil.

\section{OBJETIVOS}

Traduzir o guia Convexity Assessment Guide para a língua portuguesa. Realizar a validação semântica, linguística, conceitual e cultural do Guia de Convexidade.

\section{MÉTODOS}

Trata-se de estudo metodológico de tradução, descritivo qualitativo, cujos procedimentos metodológicos seguidos foram as fases preconizadas para essa metodologia: tradução, tradução de volta à língua de origem (back-translation), comparação das versões originais e do back-translation, validação da tradução por comitê nacional de especialistas, sendo realizado em 2015 e 2016.

Quanto aos aspectos éticos, esta pesquisa foi a fase inicial do projeto de dissertação de mestrado em enfermagem. 
A utilização e a tradução do Convexity Assessment Guide foram autorizadas pela Hollister Incorporated, Hollister do Brasil e pelos principais autores, detentores da patente do instrumento. A participação dos enfermeiros e tradutores só foi iniciada após preenchimento do termo de consentimento livre e esclarecido. Foram respeitados os regulamentos éticos de pesquisas envolvendo seres humanos. O estudo foi aprovado pelo comitê de ética e pesquisa (parecer 1.027.989) e foram observados os preceitos éticos preconizados na Resolução No 466 do Conselho Nacional de Saúde ${ }^{12}$.

\section{Adaptação do Convexity Assessment Guide para a língua portuguesa}

A tradução do Convexity Assessment Guide foi realizada em três etapas: tradução, tradução de volta à língua de origem (back translation) e validação da tradução pelo comitê de especialistas. Foram adotadas apenas essas etapas pelo fato de a convexidade ainda ser pouco estudada no Brasil, necessitando maior entendimento geral a respeito do tema para ampliar o processo de tradução para validação transcultural do guia.

\section{Etapa 1. Tradução}

O guia, na sua versão original em inglês (VI1), foi traduzido por dois tradutores independentes, originando duas versões em português (VP1) e (VP2); em seguida, foram comparadas por três enfermeiras especialistas e fluentes na língua inglesa (E1) (duas estomaterapeutas e uma mestre em enfermagem), compilando as VP1 e VP2 e gerando a primeira versão completa em português (VP3).

\section{Etapa 2. Tradução de volta à língua de origem (back-translation)}

A VP3 foi encaminhada para dois tradutores/ professores na língua inglesa e portuguesa, não informados do objetivo da pesquisa, para realização da tradução reversa do guia, sendo a VP3 traduzida do português para o inglês, gerando a versão em inglês 2 (VI2) e a versão em inglês 3 (VI3).

$\mathrm{Na}$ sequência, um grupo de quatro enfermeiras estomaterapeutas com mestrado e/ou doutorado na área (E2) compararam as duas traduções reversas (VI2 e VI3) com a versão original (VI1), no intuito de adequar quanto à semântica, ao conceito e à cultura brasileira, gerando, assim, a versão em português 4 (VP4).

\section{Etapa 3. Validação da tradução pelo comitê de especialistas}

A VP4 foi submetida na forma de instrumento de avaliação de concordância semântica e linguística a um grupo de 10 enfermeiros estomaterapeutas para verificação do nível de concordância com as questões descritas. Por se tratar de estudo de tradução, mesmo que a fase metodológica da qual o grupo participou tenha exigido conhecimentos da língua inglesa, 40\% de seus participantes declararam ter conhecimento em inglês básico, $40 \%$ intermediário e $20 \%$ avançado. Essa heterogeneidade do grupo promove uma linguagem técnica com amplitude de entendimento por diversas culturas e confere o caráter nacional à validação do instrumento.

A avaliação semântica do conteúdo se refere à análise do significado de cada frase e se as frases condizem com a realidade específica na estomaterapia. Foi utilizada a escala de Likert ${ }^{13}$ com pontuação de 1 a 5 , na qual 1 representa "discordo fortemente", 2 "discordo", 3 "não concordo nem discordo", 4 "concordo" e 5 "concordo fortemente" para classificarem a concordância semântica e linguística dos itens. O nível de concordância aceitável para validar cada item foi de, no mínimo, 90\% entre as opiniões dos estomaterapeutas.

Nos casos nos quais a pontuação representou alguma discordância (pontuação 1, 2 ou 3), o enfermeiro indicou uma sugestão de mudança para o item. A versão em português 5 (VP5) foi o resultado das alterações sugeridas e de todos os itens validados com concordância de no mínimo $90 \%{ }^{14}$ entre o grupo. Para verificar as características culturais e conceituais, alguns itens puderam ser incluídos ou retirados do instrumento.

O tratamento estatístico dos dados deu-se por meio do programa Windows Excel 2010 com análise dos níveis de concordância, utilizando porcentagens baseadas no total das respostas válidas. Foram adotados os critérios de exclusão na fase de validação, como indicações de alterações relacionadas a outros aspectos, respostas em branco, indicações de alterações estéticas e/ou não relacionadas à avaliação semântica, linguística, cultural e conceitual. Após a aplicação desse filtro, o tratamento estatístico teve seu total baseado apenas nos dados 
válidos, sendo os nulos subtraídos do total geral. Os números serão apresentados com duas casas decimais e em porcentagem nas formas de tabelas e figuras.

\section{RESULTADOS}

Em sua versão original, a parte teórica introdutória foi traduzida do inglês para o português e submetida à análise do trio de enfermeiras especialistas com vasta experiência clínica na área de assistência à pessoa com estomia, sendo duas estomaterapeutas e uma mestre em enfermagem e especialista em educação, todas fluentes na língua inglesa. Obteve-se grau de concordância de 100\% nas traduções das características chaves para a avaliação das estomias propostas pelo guia (Tabela 1). Em seguida, o guia apresenta a ficha de avaliação com 40 frases/itens submetidos ao processo de tradução em três etapas.

A ficha de avaliação é composta por 40 frases/itens de avaliação referentes à identificação do perfil do paciente, tipo de estomia, características do efluente, da estomia e do plano anatômico abdominal, assim como identificação de algumas complicações periestomais. A ficha incluiu campos para anotação dos níveis de concordância de cada frase.

O processo de tradução da ficha de avaliação da versão original VI1 para as primeiras versões na língua portuguesa VP1 e VP2 não apresentou diferenças significativas entre os tradutores, sendo, na sequência, avaliado pelo trio de enfermeiras, gerando concordância acima de $90 \%$; assim, as versões VP1 e VP2 foram consolidadas na terceira versão em português, a VP3.

Para as duas traduções reversas do português para o inglês da versão VP3, não houve diferença significativa entre os outros dois tradutores e foram analisadas pelo mesmo trio de enfermeiras da primeira etapa, ficando o grupo acrescido de uma enfermeira doutora e especialista nos cuidados com a pele, sendo também fluente em inglês. O grupo não identificou discrepâncias entre as traduções, compilando a VI2 e VI3 e validando a VP3. Nessa fase, o quarteto de especialistas também realizou a inclusão do item “confecção da estomia” na VP3, fazendo alusão às estomias em alça ou estomias terminais, item que não existia no VI1, com intuito de realizar a validação cultural e conceitual.

Para o processo de validação semântica e linguística, reuniu-se um grupo de 10 enfermeiros composto por nove mulheres, de diversas regiões do Brasil, sendo 30\% do interior de São Paulo, 30\% da cidade de São Paulo, 20\% do estado de Minas Gerais, 10\% do estado do Paraná e $10 \%$ do estado da Bahia. Quanto à formação acadêmica, nove são especialistas em estomaterapia e uma especialista em educação em saúde, $50 \%$ mestres e $20 \%$ doutores. Os profissionais foram selecionados e convidados a participar da pesquisa pela sua atuação profissional, atendimento a pacientes com estomias e conhecimento a respeito do tema, e por atuarem em regiões com grande número de pessoas com estomias.

Com relação à familiaridade, todos os enfermeiros atendem pacientes com estomias em sua rotina de trabalho, sendo $60 \%$ atuantes em ambulatórios especializados em estomaterapia com média de 35 atendimentos semanais, $10 \%$ em unidade hospitalar especializada em estomaterapia, 30\% em educação de profissionais e pacientes.

$\mathrm{Na}$ classificação dos 40 itens, obtiveram-se 400 avaliações. Após a aplicação dos critérios de exclusão, anularam-se 49 avaliações, restando 351 classificações válidas que representaram o total utilizado para a análise de concordância de cada item (Figura 1).

Tabela 1. Descrição de algumas características para avaliação das estomias de acordo com Convexity Assessment Guide.

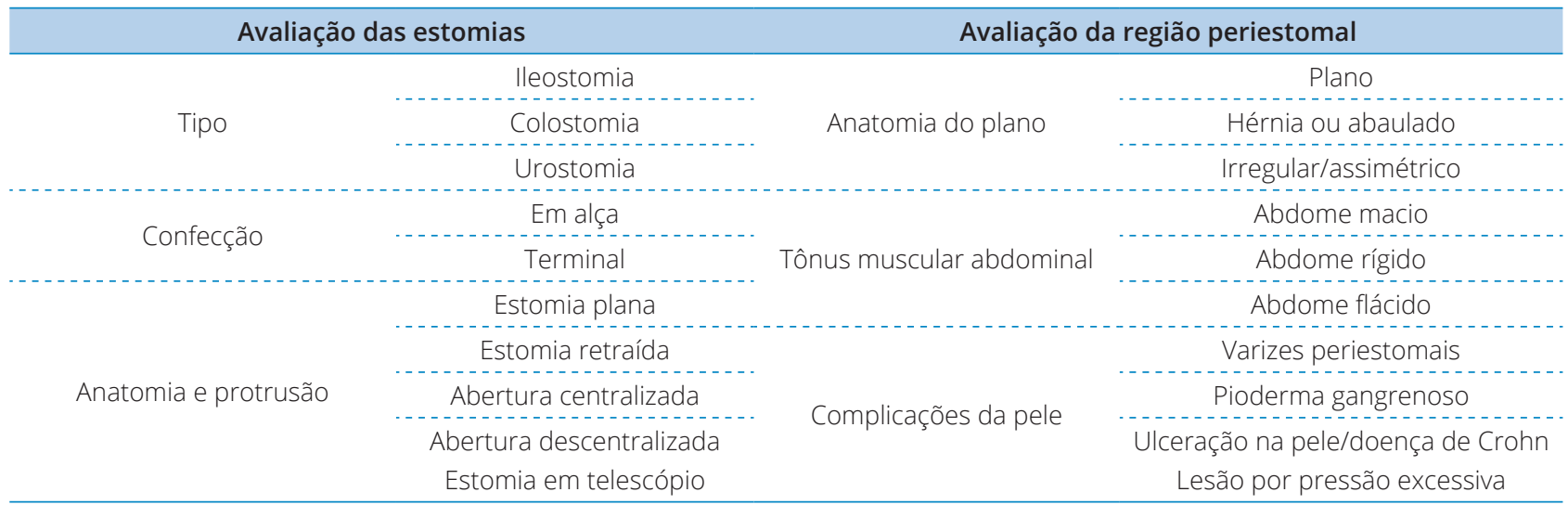


Para a classificação de cada item, o instrumento de coleta seguiu a divisão de temas proposta pelo guia original contendo as seguintes sessões: Identificação do paciente; Avaliação da Estomia - para estomias terminais e/ou em alça; Avaliação da estomia distal - apenas para estomias em alça; Avaliação periestomal - para todos os tipos de estomias; e Resultado da avaliação. Após a análise de concordância de cada um dos 40 itens, apenas seis apresentaram concordância menor de $90 \%$ entre as respostas, um item com 89\% de concordância na sessão Identificação do Paciente, nesse caso, um item foi incluído pelo comitê avaliador, dois itens na sessão Avaliação da estomia - para estomias terminais e/ou em alça, com 87 e 89\%, três itens da sessão Resultado da avaliação apresentaram classificações de $87 \%$ para um item e $89 \%$ para os outros dois (Tabela 2).

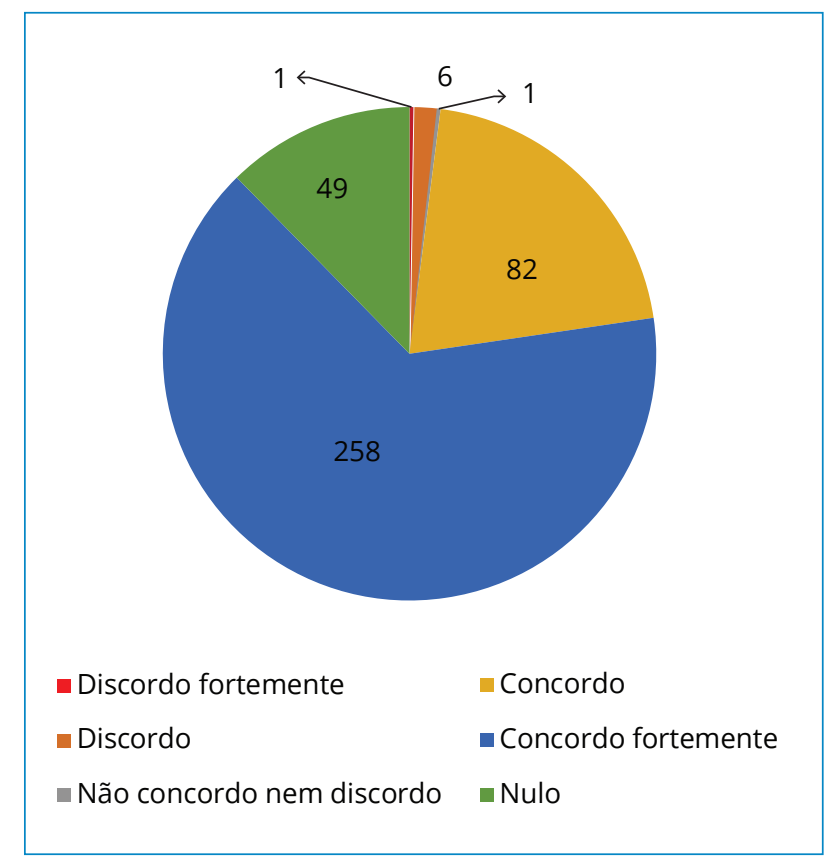

Figura 1. Representação gráfica da quantificação dos graus de concordância do instrumento de validação semântica $(n=400)$.
Os itens em discordância receberam seis sugestões para adequação de cada frase para a língua portuguesa, visando à linguagem técnica. Essas propostas foram analisadas pela autora e pelo grupo de enfermeiras E2 e, após discussão e pertinência, foram acatadas ou não, realizadas as adequações, alcançando os níveis de concordância necessários para a validação da tradução.

\section{DISCUSSÃO}

O processo de tradução e validação de instrumentos para a língua portuguesa vem sendo amplamente utilizado no Brasil em diversos setores da área da saúde, devido à complexidade que envolve a elaboração e validação de um instrumento, situação que se complica quando é voltado à área da estomaterapia ${ }^{6}$. Essa especialidade iniciou sua expansão na década de 1990, com a criação da Associação Brasileira de Estomaterapia (Sobest), entidade que apoia os estomaterapeutas do Brasil, promovendo eventos, cursos e desenvolvimento de conhecimento científico na área ${ }^{15}$.

O grupo predominantemente feminino de estomaterapeutas participantes da pesquisa - 10\% (um) masculino e $90 \%$ (nove) feminino - vai ao encontro com o demonstrado nas pesquisas relacionadas à investigação de gênero entre os profissionais da enfermagem no Brasil e, consequentemente, na estomaterapia, uma vez que a profissão do enfermeiro é historicamente feminina ${ }^{15}$.

Quanto à familiaridade com o cuidado com estomias, o grupo mostrou-se altamente envolvido com os cuidados técnicos, assistenciais e gerenciais, uma vez que $90 \%$ atuam diretamente na área. Essa característica assemelha-se com o demonstrado em estudos a respeito dos egressos em curso de estomaterapia do interior de São Paulo, no qual 93,3\%

Tabela 2. Apresentação dos resultados da validação dos itens em discordância. São José do Rio Preto/SP, 2017.

\begin{tabular}{|c|c|c|c|}
\hline Versão original & Antes da validação (VP4) & Sugestões para validação & Após a validação (VP5) \\
\hline Não contém & Construção da estomia & $\begin{array}{l}\text { Confecção da } \\
\text { estomia }\end{array}$ & Confecção da estomia \\
\hline $\begin{array}{c}\text { What type of output does the } \\
\text { patient have? }\end{array}$ & Qual tipo de efluente? & Qual o tipo do efluente? & Qual o tipo do efluente? \\
\hline Where is the stoma opening? & $\begin{array}{l}\text { Onde é a abertura da } \\
\text { estomia? }\end{array}$ & $\begin{array}{l}\text { Onde é a abertura de } \\
\text { drenagem da estomia? }\end{array}$ & $\begin{array}{c}\text { Onde é a abertura da } \\
\text { estomia? }\end{array}$ \\
\hline Assessment score indicator & Score da avaliação & Resultado da avaliação & Resultado da avaliação \\
\hline Current products used & Produtos usados atualmente & $\begin{array}{l}\text { Equipamentos usados } \\
\text { atualmente }\end{array}$ & Produtos usados atualmente \\
\hline Products recommended & Produtos recomendados & Equipamentos recomendados & Produtos recomendados \\
\hline
\end{tabular}

VP4 = versão em português 4; VP5 = versão em português 5. 
dos estudantes da pós-graduação já trabalhavam na área antes de ingressarem no curso ${ }^{15}$. Esses dados demonstram o interesse dessa classe em transformar seus conhecimentos práticos e empíricos em científicos e assertivos, mesmo comportamento também identificado em todas as etapas desta pesquisa.

O fato de haver poucos itens em discordância, apenas $17,5 \%$ de todos os itens avaliados, demonstra que a linguagem utilizada está de acordo com o utilizado regularmente, porém as discordâncias representaram importante ponto de discussão, já que a linguagem específica na estomaterapia vem evoluindo ainda de forma tímida, com alguns estudos que sugerem a padronização da linguagem e sistematização da assistência pré- e pós-operatória ${ }^{4,9,16}$.

Por escolha do primeiro trio de enfermeiros (E1) levantou-se a necessidade de se identificar, no próprio instrumento, o tipo de confecção da estomia, se terminal ou em alça. Por se tratar de um guia que deverá ser utilizado por enfermeiros especialistas e não especialistas, deve conter todas as informações prioritárias, como a que foi acrescida e validada pelo comitê de especialistas. Mesmo estando entre os itens em discordância, as sugestões foram extremamente válidas, sendo aprovadas pelo grupo, uma vez que estudo realizado recentemente comprova que diversos fatores associados à confecção da estomia podem levar ao desenvolvimento de complicações periestomais ${ }^{16}$.

As discordâncias e os desconfortos ligados ao item "tipo do efluente" foram amplamente discutidos, devido à importância em caracterizar e mensurar o volume do efluente, pois a principal complicação apresentada por pessoas com estomias, a dermatite periestomal, pode ser causada pelo contato direto do efluente com a pele ${ }^{8}$. Esse tipo de lesão é classificado como lesão periestomal associada à umidade, por consenso de $2013^{17}$. Assim como a caracterização do efluente se faz importante para a escolha do equipamento ideal para o paciente, a identificação da abertura da estomia é crucial, pois sua posição direciona o fluxo do efluente podendo, se descentralizada ou em telescópio, facilitar a infiltração do efluente por baixo da barreira adesiva da bolsa coletora ${ }^{18}$.

Durante a validação, foi sugerido, por $20 \%$ dos participantes, que a palavra "escore", de origem inglesa, fosse substituída por "resultado", justamente por ser um guia de aplicação nacional e na língua portuguesa. Mesmo sendo a palavra "escore" amplamente utilizada no Brasil, as sugestões foram aceitas e validadas pelo grupo ${ }^{19}$. Estudo de tradução de instrumento na área de ortopedia manteve o uso original da palavra score apenas adaptando para a linguagem portuguesa "escore", ficando evidente a liberdade de escolha e validação do termo utilizado para cada classe de profissionais; nesse sentido, acatou-se a utilização do termo "resultado", validado com $90 \%$ de concordância.

Os dois últimos itens em discordância também apresentaram sugestões análogas. Devido à semelhança entre as frases, acatou-se a sugestão do comitê de especialistas, por se tratar da anotação e indicação de diversos "produtos" relacionados ao cuidado com a estomia, englobando o "equipamento" coletor e outros adjuvantes que auxiliam no melhor ajuste desse à estomia, não apenas o equipamento ${ }^{20}$. Identificou-se, em estudo recente com enfermeiros estomaterapeutas norte-americanas, que as principais escolhas de produtos para o manejo de complicações periestomais envolvem equipamentos coletores com base convexa entre outros adjuvantes ${ }^{21}$. Essas indicações corroboram a escolha da terminologia adotada na versão final do guia. A indicação adequada dos equipamentos, $o$ atendimento de uma enfermeira estomaterapeuta e o apoio familiar são imprescindíveis para o desenvolvimento do autocuidado ${ }^{22}$, uma vez que a complicação de pele periestomal mais comum é resultado do contato do efluente com a pele, causado pela falta de ajuste adequado do tipo de barreira com o tipo de pele e de estomia ${ }^{23}$. A garantia da saúde da pele periestomal promove qualidade de vida àquele que vive com estomia ${ }^{23}$.

\section{Limitações do estudo}

As principais limitações do estudo ocorreram durante o levantamento bibliográfico, devido à escassez de produção científica nacional a respeito do uso de convexidade em estomias e à dificuldade em recrutar enfermeiros estomaterapeutas com conhecimento avançado e fluência na língua inglesa.

\section{Contribuições para a área de enfermagem}

Este estudo promove a primeira evidência científica relacionada à tradução de instrumentos que auxiliam na indicação clínica de dispositivos coletores para estomias de eliminação. Obteve-se, por meio desta pesquisa, a validação da tradução do Convexity Assessment Guide para a língua portuguesa. 


\section{CONCLUSÃO}

Nessa fase de tradução, validação semântica, linguística, conceitual e cultural do Guia de Convexidade, verificou-se alto nível de concordância do comitê de avaliadores, garantindo a continuidade do estudo com a validação transcultural do Guia de Convexidade para a realidade brasileira.

Este estudo recebeu o apoio da Hollister do Brasil e Hollister Incorporated.

\section{CONTRIBUIÇÃO DOS AUTORES}

\author{
Conceitualização, Rafaldini BP e Poletti NAA;
} Metodologia, Rafaldini BP; Poletti NA e Ruiz PBO; Investigação, Rafaldini BP; Ruiz PBO e Squizatto RH; Redação - Primeira versão, Rafaldini BP; Redação Revisão \& Edição, Rafaldini BP; Lopes $A O$ e de Oliveira NC; Recursos, Rafaldini BP; Supervisão, Poletti NAA.

\section{REFERÊNCIAS}

1. Wound Ostomy and Continence Nurses Society. Scientific and Clinical Abstracts from the 2016 WOCN Society \& CAET Joint Conference; 2016 Jun 4-8; Montreal, Canada. Disponível em: https://journals.Iww.com/jwocnonline/pages/default.aspx

2. World Council Enterostomal Therapists. International Ostomy Guideline. [citado 2016 Set 01]. Disponível em: http://www. wcetn.org/international-ostomy-guideline

3. Sociedade Brasileira de Estomaterapia. Anais do XI Congresso Brasileiro de Estomaterapia; 2015 Nov 1-4; Gramado, Brasil. Disponível em: http://sobest.org.br/publicacoes/cbe-15/anais

4. Haugen $V$, Ratliff CR. Tools for assessing peristomal skin complications. J Wound Continence Nurs. 2013;40(2):131-4. https://doi.org/ 10.1097/WON.0b013e31828001a7

5. Giusti E, Befi-Lopes DM. Tradução e adaptação transcultural de instrumentos estrangeiros para o Português brasileiro (PB). Pró-Fono R Atual Científica. 2008;20(3):207-10.

6. Domansky RC, Santos VLCG. Adaptação cultural e validação do instrumento The Bowel Function in the Community para o Brasil. Rev Esc Enferm USP. 2009;43(Espec):1114-29.

7. Miranda SM, Luz MHBA, Sonobe HM, Andrade EMLR, Moura ECC. Caracterização sociodemográfica e clínica de pessoas com estoma em Teresina. ESTIMA, Braz J Enterostomal Ther. 2016;14(1):29-35. https://doi.org/10.5327/Z18063144201600010005

8. Salvadalena DG. The incidence of stomal and peristomal complications during the first 3 months after ostomy creation. J Wound Ostomy Continence Nurs. 2013;40(4):400-6. https:// doi.org/ 10.1097/WON.0b013e318295a12b

9. Hoeflok J, Kittscha J, Purnell P. Use of convexity in pouching. A comprehensive review. J Wound Continence Nurs. 2012;40(5):506-12. https://doi.org/10.1097/ WON.0b013e3182a219b7

10. Nail N, Inlgese G, Manson A, Townshend A. A cost-utility model of care for periestomal skin complications. J Wound Ostomy Continence Nurs. 2016;43(1):62-8. https://doi.org/ 10.1097/WON.0000000000000194

11. Robertson JP, Wells $\mathrm{Cl}$, Vather R, Bossett IP. Effect of diversion ileostomy on the occurrence and consequences of chemotherapy-induced diarrhea. Dis Colon Rectum. 2016;59(3):194-200. https://doi.org/10.1097/ DCR.0000000000000531

12. Ministério da Saúde (BR). Conselho Nacional de Saúde. Resolução No 466, de 12 de dezembro de 2012. [Internet].
Brasília, DF; 2012. Disponível em: http://bvsms.saude.gov.br/ bvs/saudelegis/cns/2013/res0466 1212 2012.html

13. Pasquali L. Instrumentação psicológica. Fundamentos e práticas. Porto Alegre: Artmed: 2010.

14. Tilden VP, Nelson CA, May BA. Use of qualitative methods to enhance content validity. Nurs Res. 1990;39(3):172-5.

15. Dias MSC, Paula MAB, Morita ABPS. Perfil profissional de enfermeiros estomaterapeutas egressos da Universidade de Taubaté. ESTIMA, Braz J Enterostomal Ther. 2014;12(3).

16. Silva ES, Castro DS, Garcia TR, Romero WG, Primo CC. Tecnologia do cuidado à pessoa com colostomia: diagnósticos e intervenções de enfermagem. REME - Rev Min Enferm. 2016;20:e931. https://doi.org/10.5935/1415-2762.20160001

17. Gray M, Colwell JC, Doughty D, Goldberg M, Hoeflik J, Manson A et al. Peristomal moisture-associated skin damage in adults with fecal ostomies: a comprehensive review and consensus J Wound Continence Nurs. 2013;40(4):389-99. https://doi. org/10.1097/WON.0b013e3182944340

18. Ratliff CR. Factors related to ostomy leakage in the community setting. J Wound Ostomy Continence Nurs. 2014;41(3):24953. https://doi.org/10.1097/WON.0000000000000017

19. Silva ALP, Croci AT, Gobbi RG, Hinckel BB, Pecora JR, Demange MK.. Tradução e validação da nova versão da escala Knee Society Score - The 2011 KS Score - para a língua portuguesa. Rev Bras Ortop. 2017;52(4):506-10. https://doi.org/10.1016/j. rbo.2016.08.005

20. Stelton S, Zulkowski K, Ayello EA. Practice implications for periestomal skin assessment and care from 2014 World Concil of Enterostomal Therapists. International Ostomy Guideline. Adv Skin Wound Care. 2015;28(6):275-84. https:// doi.org/10.1097/01.ASW.0000465374.42350.0f

21. Beitz JM, Colwel JC. Management approaches to stomal and periestomal complications. A narrative descriptive study. J Wound Continence Nurs. 2016;43(3)263-268. https://doi. org/10.1097/WON.0000000000000215

22. Luo B-J, Zheng M-C, Zhang J-E, Jiang M-X, Pan Z-Z et al. Stoma self-efficacy, hope and social relationships among patients with permanent colostomy. WCET journal. 2018;38(1):12-20.

23. Colwell JC, Pittman J, Raizman R, Salvadalena G. A randomized controlled trial determining variances in ostomy skin conditions and the economic impact (ADVOCATE Trial). J Wound Continence Nurs. 2018;45(1):37-42. https://doi.org/ 10.1097/WON.0000000000000389 